\title{
ORGANIC USER INTERFACES: FRAMEWORK, INTERACTION MODEL AND DESIGN GUIDELINES
}

\author{
Prof. Dr. Atef Zaki Ghalwash and Sara Khaled Nabil \\ Computer Science Department Helwan University Cairo, Egypt
}

\begin{abstract}
Under the umbrella of Ubiquitous Computing, lies the fields of natural, organic and tangible user interfaces. Although some work have been made in organic user interface (OUI) design principles, no formalized framework have been set for OUIs and their interaction model, or design-specific guidelines, as far as we know. In this paper we propose an OUI framework by which we deduced the developed interaction model for organic systems design. Moreover we recommended three main design principles for OUI design, in addition to a set of design-specific guidelines for each type of our interaction model.

Our proposed framework is deduced based on previous work of other related researches for Graphical User Interface (GUI) and Tangible User Interface (TUI) frameworks. By categorizing the input techniques of OUI systems, we were able to propose the '/ Manipulation/ air-Gesture' (SMaG) interaction model. Each category of SMaG model is explored thoroughly and criticized accordingly. Based on the SMaG interaction model we introduced some design guidelines for OUIs. The proposed OUI design principles are based on three usability aspects: look, feel and design. We conclude by pointing out our proposed OUI usability guidelines and considerations regarding design-specific organic interfaces that uses each of the input interaction techniques of SMaG model, their best use, worst use and how to use.
\end{abstract}

\section{KEYWORDS}

Organic User Interface, design guidelines, tangible interfaces, interaction techniques, gestures.

\section{INTRODUCTION}

After decades of traditional desktop computers that are used in specific contexts such as education, scientific research, heath care, industry, information technology, ..etc by expert users that are professional at dealing with computer devices through typing predefined sequential text commands or performing specific actions for each required task by running set of programs or processes, after all that today computers have become in every hand and the technology has enfolded a much wider meaning that it used to have any time before. Technology is heading towards context-aware ubiquitous environments where the target is now the normal user and the contexts has become the daily actions not just the different professional fields of health, education, military and other fields of technology.

DOI : $10.5121 /$ ijasuc.2013.4404 
Ubiquitous Computing includes novel forms of devices and interaction techniques for interfaces that are natural, tangible and organic user interfaces. Natural user interface (NUI) is the type of interfaces with no traditional means of data input, but natural actions and interaction techniques that opens the way for ubiquitous multi-touch multi-user computers. Since the rise of NUIs no more separated input devices such as mice are needed. Examples of NUIs are today's tablets, multitouch smart phones such as iPhones. Some even may consider the Microsoft Surface and The Sphere to be NUI systems as well, although they might be considered OUI systems.

Tangible user interface (TUI) -subset of NUI- is the type of interfaces that uses graspable physical artifacts- called tangibles- to control digital information seamlessly in the user's physical environment. Thus, tangible interfaces make use of the already existing usability experience and haptic interaction skills that users have with real-world objects. Not only does TUI give physical representation to the digital content, but also enables this digital content -represented by the physical tangible- to be directed manipulated through the user by his bare hand. TUI's interaction style and physical representation of data -rather than graphical representation- is the main advantage for users and their perception for the user interface over GUI.

Organic User Interface (OUI) is not just a subset of NUI, but the legal heir of NUI if we might say so, as per Dennis Wixon who suggested that OUI will be the successor of NUI moving the leverage of user interfaces from relationships -in natural interfaces- to special memory in organic interfaces benefiting from the new organic technologies that enables flexible display materials and flexible paper-like electronics.

In 2008, Raol Vertagaal and Ivan Poupyrev [1] defined OUI as interfaces that have non-planar displays that may actively or passively change shape via analog physical inputs. Organic displays are characterized by two main factors: they can change or take on any shape and they can be used as an input device.

The term 'organic' came from four facts:

1. First, these new flexible interfaces are built from the newly developed organic electronics and materials.

2. Second, the malleable form and flexible shape of OUI systems make give them an 'organic look'.

3. Third, the dynamic structure of OUI devices enables them to give visual feedback by changing their physical shape actively or passively which gives them an 'organic feel' as if the device is alive.

4. Forth, the human-computer interaction in an OUI system is more likely similar to humanhuman interaction resembling the natural human-physical interaction[2] such as grabbing, folding, shaking and many other gestures.

This organic uprising technology that is starting to emerge today lacks the standards and guidelines that controls and facilitates the design process of OUI devices, in comparison with the efforts previously exerted in the GUI design. As far as we know, no clear and detailed design principles have been offered for organic computers design, other than the three principles of OUI design presented in [3]: Input Equals Output, Function Equals Form, and Form Follows Flow. Moreover, Holman et al. in "Organic User Interfaces" introduced four broad rules that must be followed by OUI designers: Fluidity, intuitiveness, Robustness and Calmness. 
However, the fact that digital devices are to be designed in organic shapes in the near future, puts designers in need for a strong solid base of principles that guide the design of organic devices and its usability. Although some work have been made in OUI design principles, no framework have been set for OUIs and their interaction model.

In this paper we will propose an interaction framework for OUIs based on its predecessors TUI and GUI, in addition to the input interaction technique model, design principles and usability guidelines for organic interfaces. The paper is organized as following: Section $I$ is the Introduction, Section II is the related work survey for the previous frameworks introduced for previous user interfaces and the related work in OUI design principles. Section III introduces our proposed framework based on the previous related work, Section IV presents and explains our proposed OUI input-interaction 'SMaG' model, and finally Section V presents our proposed OUI design guidelines based on the SMaG model. Last, comes the conclusion, future work and references.

\section{RELATED WORK}

\section{A. GUI \& TUI Frameworks}

The related work we studied for user interfaces frameworks included papers[1,4] for TUI framework as an evolution to its predecessor GUI framework. However no work -as far as we know- was found that contributes to OUI framework, that a proposal is introduced in this paper. In 2001, Ullmer and Ishii in [4] proposed a framework for Tangible User Interface (TUI) in comparison with Graphical User Interface (GUI) framework. The GUI framework is constructed from the MVC model (Model-View-Control) where there is a clear distinction between the input and the output devices that are completely separated from each other in the physical layer, as seen in the figure 1.

The TUI framework developed the MVC model to MCRpd: Model-Control-Representation (physical and digital). MCRpd framework keeps the 'model' in the digital layer, the 'view' however, is divided into both a physical representation and a digital representation denoted by 'rep-p' and 'rep-d' respectively. This framework demonstrates the two types of digital information representation in the physical layer, one that is graspable (physical objects), and one that is non-graspable (such as sound, video projection, ..etc) 


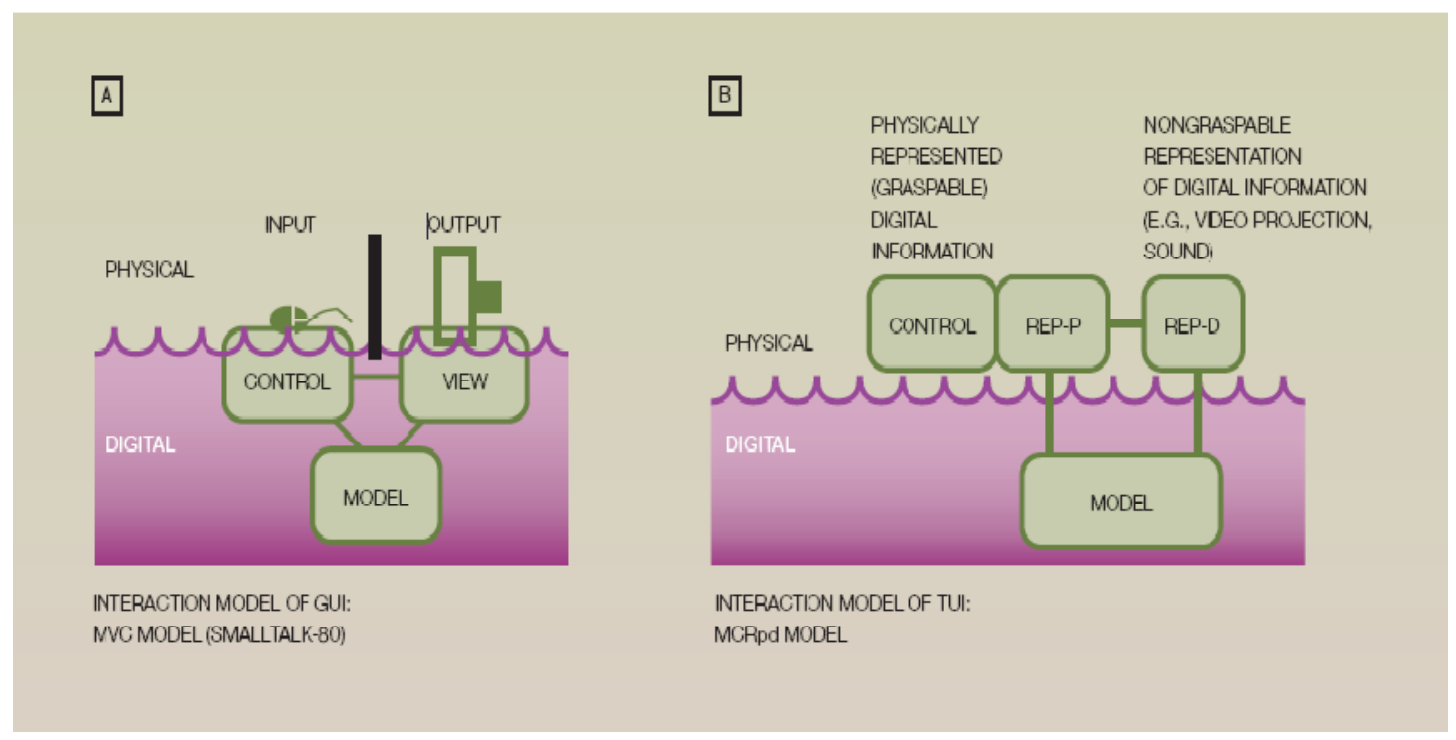

Figure 1 : MVC model vs. MCRpd model[4]

In 2008, Hiroshi Ishii[1] enhanced this TUI framework, highlighting there is no more separation between the input and output devices as was in the previous GUI framework (MVC model). Ishii's framework also presents the graspable and in-graspable (tangible and in-tangible) digital information (rep-p and rep-d) as tangible representation and intangible representation, representations of both physical and digital input interaction styles, as shown in figure 1.

\section{B. WIMP and Post-WIMP Interaction models}

After decades of WIMP interfaces (window-icon-menu-pointer) based on the GUI framework, post-WIMP interfaces are rising intensively promising a new era of user-input interaction styles for NUI (natural user interface), TUI and OUI. WIMP interactions rely on the technology created by the colored screen monitor controlled by the keyboard and mouse input devices, depending mainly on graphics. With new natural, tangible and organic interfaces evolving, and by the gradual disappear of keyboards and mice, WIMP techniques can no longer be used as is. Thus, there is a certain need for new interaction techniques that can provide the user with seamless intuitive natural experience when interfacing in such ubiquitous environments. We would need rather more continuous input techniques that have more natural interactions than the discrete input interactions of the earlier UIs: GUI and CLI. In [5] Van Dam named this new generation of interactions the post-WIMP interfaces.

Worth mentioning as well, is that augmented or virtual reality widgets are considered post-wimp interfaces, were virtuality systems make use of tangibles such as head mounted displays, gloves and 3D caves as interactive post-wimp input and output devices.

Post-WIMP interfaces are already employed in computer video games using physical tangibles such as a car wheel and gas and breaks peddles and wii remote motion controller -also called 'wiimote'- that recognizes gestures and pointing. Multi-touch interactive displays are another type of post-wimp interfaces as it no longer depends on mouse pointer or window menus. 
International Journal of Ad hoc, Sensor \& Ubiquitous Computing (IJASUC) Vol.4, No.4, August 2013

However, scientifically speaking, there is no clear aspects of post-WIMP interaction style that define it clearly except that it depends on multitouch displays, human gestures and perhaps speech recognition as well.

\section{OUI Design principles and guidelines}

In spite of the intensive studies in the field of GUI design principles, there have been unfortunately- only quite a few attempts to set the rules and principles for OUI design.

Holman and Vertegaal[3] presented the three main principles of OUI design. These three principles are the basic foundation and building blocks for any OUI design guidelines or rules.

\section{Input Equals Output}

(When display is the input device)

This design principle states that in organic computers the output displays could also act as input devices. For example: OLED displays with multi-touch sensors for data input.

On contrary with GUI-based computers, OUIs merge input and output devices together. While in GUIs the input and output devices are completely separated from each other and distinct.

For example: Mouse and Keyboard (input devices) are clearly separated from monitor display(output device) in traditional point-and-click computers.

\section{Form Equals Function}

(When displays can take any form)

This design principle states that the shape or form of the Organic device should be dynamic enough so as to change to match the intended functionality.

For example: the paper computers can be bended inwards to zoom-in the content of the paper, as well as can be bended outwards to zoom-out the content.

\section{Form Follows Flow}

(When displays can change their shape)

This design principle states that the shape or form of the Organic device should be dynamic enough so as to change according to the flow of the user interactions.

For example: mobile phones change their display options

and menu while conducting a phone call while clamshells deactivates the screen while folding them.

Moreover, Holman et al. in [6] introduced four broad rules that must be followed by OUI designers. The four principles are derived from the natural laws of biology, physics and human cognition. These four principles are:

\section{Fluidity (physics laws)}

This design principle stated that the OUI is fluid when simple rules are set to enforce constraints on the system that are easily understood by the user. 
International Journal of Ad hoc, Sensor \& Ubiquitous Computing (IJASUC) Vol.4, No.4, August 2013

\section{Intuitiveness (biology laws with respect to human cognition)}

This design principle states that the OUI is a representation of the most natural interaction techniques that support the functions intended by the user, putting into consideration the limited human cognition and abilities.

\section{Robustness (biology laws)}

This design principle states that the OUI must be robust enough to easily recover from errors, operate with degraded functionality until repaired and avoids hazards.

\section{Calmness (human cognition laws)}

This design principle states that the OUI must allow the user to choose to focus on the interface when there is a need, or to lose focus when there is no need, hence, the organic system should be non-distracting in the later case.

The 'Calmness' principle is based on the fact that OUI is a subset of Ubiquitous Environments -as discussed earlier- where all computers seem to disappear in the background and nearly be unnoticeable to the user.

The drawbacks of Holman's[3] OUI design principles is that it ignores the input interaction techniques that does not necessarily equals the output. That is, for example the in-air gestures that users can use to interact with an organic device entering commands to the system to perform certain actions or apply a specific task. The same applies for speech commands that is used by several organic devices allowing hand-free control cannot be applied under the principle of "input equals output". Moreover, output does not equals input, as the system can have physical reactions to our actions -actively- or notifications -pasively- in the form of producing a sound or changing the shape or layout of the device, which does not imply that output equals input. As for the second and third principles they apply only on flexible displays that can actively or passively change their shape to allow natural input or to feedback the user with physical output. However that does not apply on all nowadays tablets and devices that enable the user to interact by touch, manipulations, speech and gestures in a combination of complete harmony using human natural abilities, postures and even eye tracking.

\section{THE PROPOSED FRAMEWORK}

The TUI framework introduced by Ishii [1] did show the distinction between two 'View' types: the tangible (graspable) and intangible (non-graspable) representation of information (physical view and digital view) denoted by rep-p and rep-d. Yet, kept the 'Control' in one single block see figure 2- with no sign of different types of user input control.

The 'control' module represents the input interaction techniques, that are also either tangible or intangible. Perhaps Ishii[1] focused on the tangible physical control only as TUIs was the scope of his research. In this paper we propose a framework for OUI based on Ishii's work, in addition we distinguish the 'tangible control' from the 'intangible control'. 


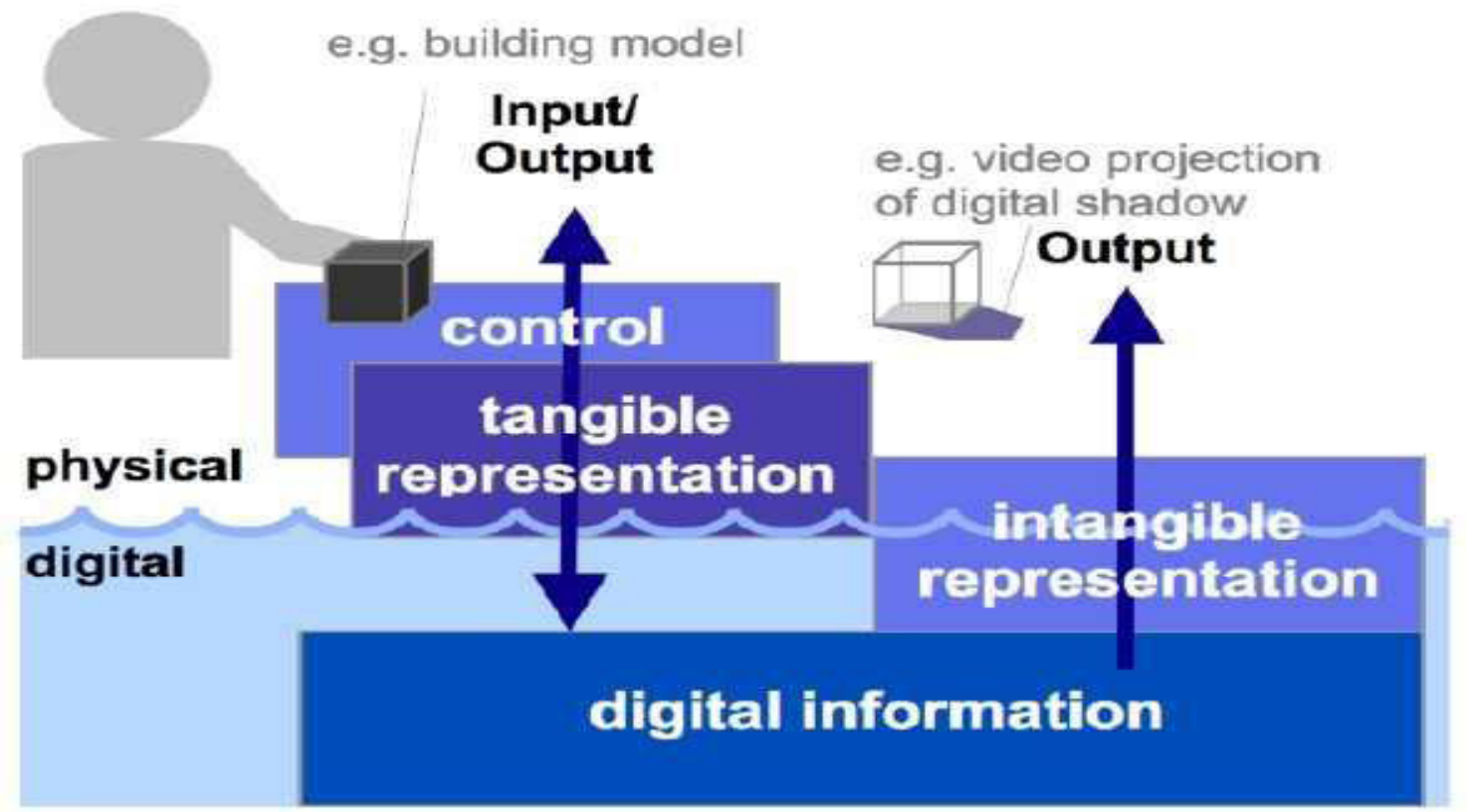

Figure 2 : Ishii's TUI Framework[1]

Moreover, we believe that our proposed OUI framework better suits the 'SMaG Interaction Model' of Organic User Interfaces, presented in this paper, based on interaction styles of speech, hand manipulations and air-Gestures used by organic systems.

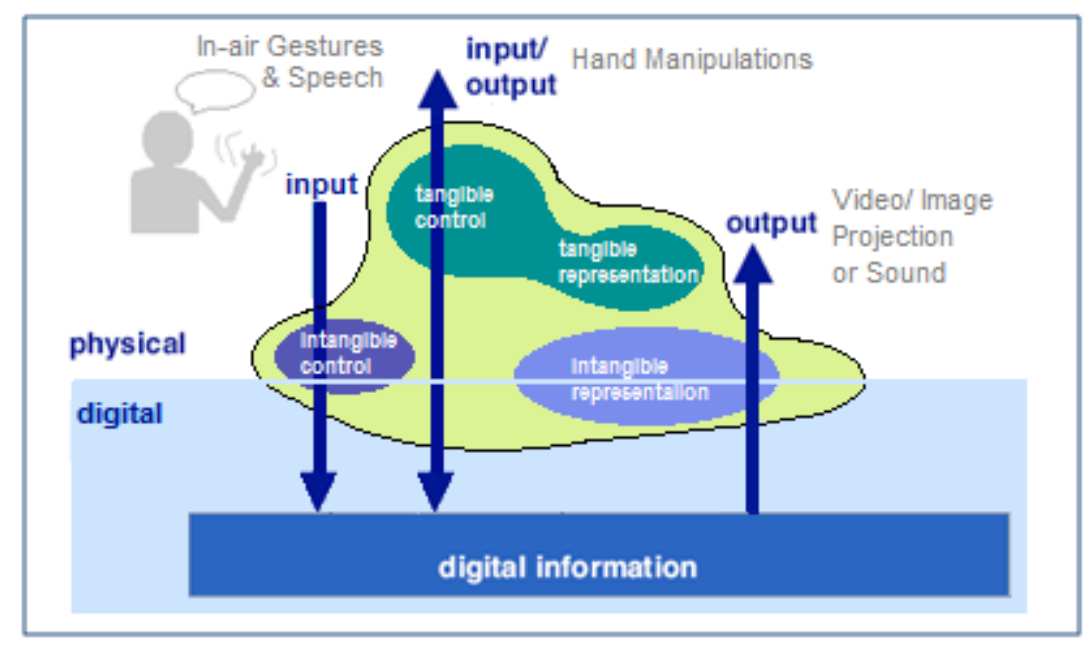

Figure 3: A proposed OUI Framework

From the fact that organic user interaction does not only depend on touch, deformation and manipulation of physical objects and tangibles, but includes in-air gestures and speech inputs as well, we divided the 'Control' into 'Tangible Control' and 'Intangible Control'. 
International Journal of Ad hoc, Sensor \& Ubiquitous Computing (IJASUC) Vol.4, No.4, August 2013

Tangible Controls are physical input interactions that the user can use while interacting with an organic system to input his required commands or actions. Physical tangible input interactions require direct contact from the user to the surface of the display or physical objects in the system. Tangible controls are mostly anticipatory, intuitive and perceived as real-world interactions. Tangible controls include hand touch, hand deformation and manipulation of displays, physical objects and tangible devices.

Intangible Controls are other SMaG model interactions, that do exist in the physical layer, but are not physically touchable or contained. Intangible interactions include speech input and in-air gestures such as hand gestures, facial expressions, body language. Intangible interactions are also natural and intuitive as tangible interactions are, however they are not anticipatory as much; they need a manual to use them when interacting with an organic system.

Tangible representation is the intersecting area between control and representation: input and output. The physical input interactions of display touch, deformation and hand manipulation is responded by the system with corresponding output interactions on the same multi-touch and/or deformable display, see figure 3. This area (tangible control and tangible representation) is consistent with the three principles of OUI design of Holman and Vertegaal's[3]. The first principle "Input Equals Output" where the display is both the input and output device, this is the area that joins the Tangible control and Tangible representation. The second principle "Form Equals Function" is where the displays can take any form during user input interaction, is the area of Tangible control. The third and last principle is "Form Follows Flow" where displays can change their shape for responsive output interaction, is the area of Tangible representation.

We can see from the above that there are interaction areas (intangible control and intangible representation) that are not included in Holman and Vertegaal's OUI design principles[3]. They did not consider that speech and/or in-air gestures can be used as interactive styles and that both of them (speech and gestures) does not necessary correspond to output response from the system. Moreover, they ignored the output interactions that may be used in an organic system that are audible or visual feedback, but not touchable, for example: producing speech or sound and image or video virtual projection on a display. Such output interactions does not require a display to change its shape or form (as the third principle states) but are still organic interactions.

\section{THE PROPOSED SMAG INTERACTION MODEL}

In [7], the author proposed the 'STaG' model for Organic Interaction in OUIs, as abbreviation for the three main categories of natural interactions: Speech, Touch and Gesture.

However, we believe that users are easily capable of performing many deformation activities that are normally 5 suitable for interacting with OUIs and are not to be considered gestures but rather 'Manipulations'. Therefore, the OUI general interaction model can be better called 'SMaG' for: Speech, Manipulations and Gestures, where Touch is considered one type of hand manipulations while Gestures are only 'in-air gestures'. 
Accordingly, we can divide the OUI user interaction techniques that we referred to in this paper as the SMaG model, to the below categorization: speech, hand manipulation and in-air gestures. Speech is divided into two subcategories: normal speech recognition and silent speech recognition for subvocal speech. Hand manipulations are either touch and tab, deformable manipulations, non-deformable manipulations, texting and drawing manipulations and manipulating tangibles or other physical objects. In-air gestures have different sub types: unimanual and bimanual hand gestures, gaze gestures (eye tracking), face gestures (facial expressions) and other body positions and movements, either for parts of the body such as arms, legs, back and shoulders or the whole body.

The SMaG categories are summarized as below:

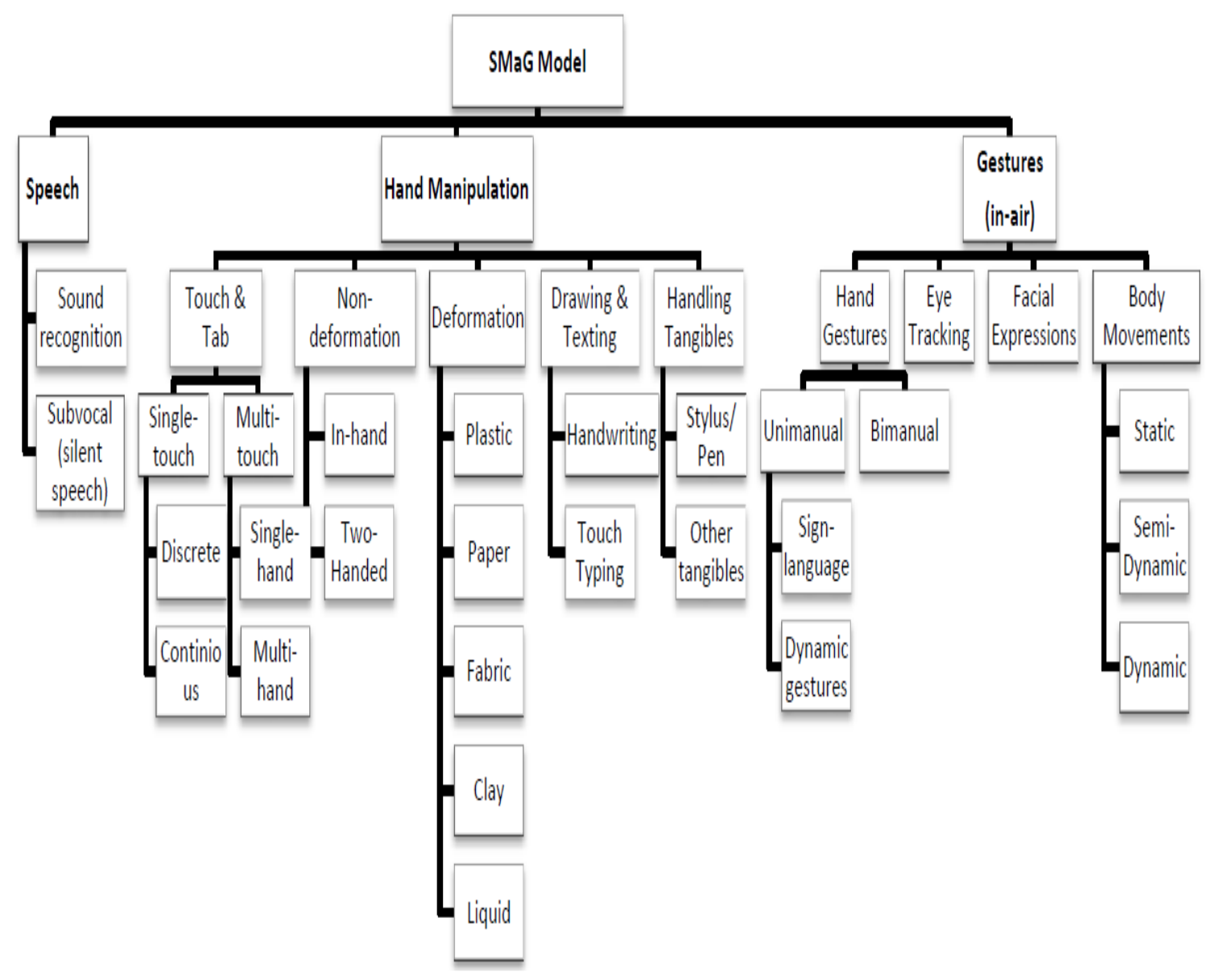

Figure 4: SMaG input interaction model for OUI

\section{A. Speech}

Speech is the oldest mean of interaction known to mankind. Moreover it requires no hand movement or body effort. As much as speech forms a natural way of communication between humans in their daily life, so it should be one day between human and computers, as much as interfaces will be more natural, and as much as computers will get ubiquitous around us. 
International Journal of Ad hoc, Sensor \& Ubiquitous Computing (IJASUC) Vol.4, No.4, August 2013

\section{1) Voice recognition:}

Speech recognition have been studied widely as an interaction technique for computer devices. Although it is proven hard to reach an optimum percent of error-free speech recognition, some systems depend on speech as an essential interaction technique specially for users with speech impairments or in medical procedures where it is a must for doctors not to compromise sterility by touching buttons, keys or switches.

However, speech interaction can be very difficult or nearly impossible in crowded or noisy places. Moreover, environments that require silent interaction for either confidentiality or specialsecurity purposes, cannot make use of normal language speech recognition technologies, but require a silent-speech recognition technology to an efficient interaction technique.

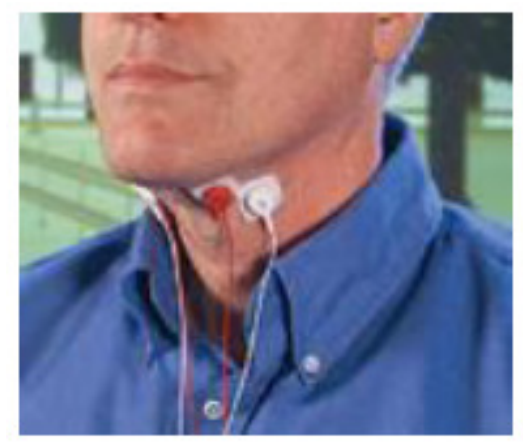

Figure 5: Subvocal Speech Interaction [8]

\section{2) Subvocal speech recognition:}

In order to overcome the limitations of using voice recognition, some researches were made to make use of the vibrations of vocal chords of a human's silent speech. Richard

Yonck in [8] described the silent speech recognition as "sub-vocal" speech recognition in which the user's silent speech forms input to the system through a small device on the user's throat reading his nerve signals directly from his throat, converting them to electric signals and sends them to a remote computer that translates them into words (commands).

As it needs no actual voice produced by the user, subvocal interaction technique can be used with speech-impaired users, firefighters and special forces. Subvocal speech can be very useful in speech non-conducting medium, such as underwater for divers or in the outer space for astronauts. NASA Ames

Research Center is currently developing a subvocal system for eventual use in astronauts' suits. [8]

\section{B. Hand Manipulation}


Hand manipulations provide users with the natural real-world intuitive interactions with computer devices through physically realistic actions. In general, hand manipulations can be divided into two types: touch, deformable and non-deformable manipulations, texting and tangible handling, motions and actions performed by the user.

\section{1) Touch \& Tab}

Using Touch input interaction style has always been an easier, faster and more fun experience for users than the use of physical input devices such as a mouse, a keyboard, a touchpad, ..etc. Moreover, being so natural and intuitive to perform, touch interactions are a very well candidate to be the primary interactive technique of tomorrow's computers.

Lately, touch interactions has been widely used in different computer devices. It is no longer a challenge to implement a touch screen instead of mechanical buttons and keys. A touch interaction can be with one or more fingers, using one, two or even more hands (in case of multiuser systems). Touch input interaction style is categorized into: Single-touch and Multi-touch. Types of touch requiring more than one finger or more than one hand is called 'multitouch'. Multitouch interaction enables the system to support multiuser interaction and collaboration. Most of the OUI systems are based on multitouch flexible organic displays.

Recently finger touch interactions have been proven to be much more than one type. In [9] researchers studied the different types of figure touch based on the fact that there are different parts in a human finger, than just the tip. The different types of finger touch introduced in [9] are the: Tip, Pad, Nail and Knuckle. These touch types presented possible different input interaction techniques, used in the Tapsense[9]. Different touch inputs can be recognized by the different effects they create, such as the touch area, shape and pressure, as seen in figure 6 .

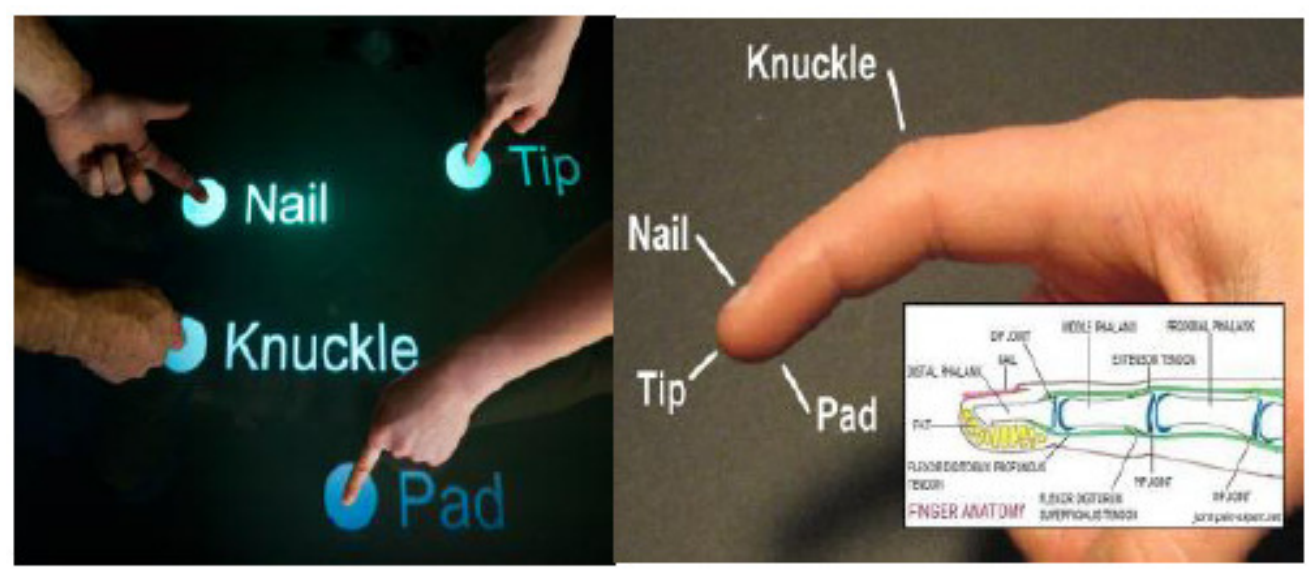

Figure 6 : Different finger Touch interactions [9]

In general, we believe that a touch input can be defined by a combination of different parameters below:

- Touch Type: tip, pad, nail, knuckle

- Number of simultaneous touch points 
- Motion of the touch or is it just a single tab

- Direction of fingers motion

- Shape formed by the touch

- Pressure pursued during the touch

- Time taken during the touch

- Distance covered by the finger on the display

- Location of contact points on the display

Tabbing is a special type of touch input and can be used by different techniques such as doubletapping (resembling double-click in previous WIMP interfaces). Tapping can be accompanied with holding or dragging to create more input commands that helps the user enter different commands to the device such as move (cut and paste) an object, zoom in and out, open, start, ..etc.

\section{2) Deformable \& Non-Deformable Manipulation}

Hand manipulations provide users with the natural real-world intuitive interactions with computer devices through physically realistic actions. In general, hand manipulations can be divided into two types: deformable and non-deformable motions and actions performed by the user.

In 2010, Sang-Su et al. in [10] studied the usability of different materials; paper, elastic cloth, and plastic by observing human gestures and behaviors of users in using deformable displays as input devices. The study included combined manipulations (such as crumpling and tearing) as well as non-deformational motions (such as rubbing, reversing and tapping).

Hand manipulation input interactions can be recognized based on three factors:[10]

i. The manipulation type

a. Deformable manipulations

b. Non-deformable manipulations

ii. The motion axis

a. Landscape: along the $\mathrm{x}$-axis

b. Portrait: along the $y$-axis

c. Diagonal: along the z-axis

iii. The motion direction

a. Positive: forward, left to right,

b. Negative: backwards, right to left

c. Dual: inwards, outwards

\section{1) Deformable Manipulation}


International Journal of Ad hoc, Sensor \& Ubiquitous Computing (IJASUC) Vol.4, No.4, August 2013

Deformable actions are input interactions that users can perform using freehand manipulations that change the shape of the deformable object or deformable display. Deformable manipulations rely on the physical human skills users already use in their real world activities. Deformation types that a user can perform using his hands depend on the deformable material itself. For example the deformations that could be done to a piece of paper are definitely different than that could be done to a piece of clay.

We divided the types of deformational hand manipulations - based on the material type- as below:

\section{i. Plastic-like}

Materials that have the same characteristics of plastic sheets can be physically piled, flipped or stacked on top of each other for browsing documents within.

\section{ii. Paper-like}

Materials that resemble real world paper can be bent, folded, wrapped, twisted, crumpled or even torn for deleting a document.

iii. Fabric-like

Elastic materials and cloth fabricated devices can be deformed to many shapes. Fabric-like materials can also be stretched, scrunched, pinched, ..etc.

iv. Clay-like

Materials that have the same feel and look of clay can be squeezed, pressed, pushed, pulled or be deformed to almost any non-regular shape.

v. Gel-like

Materials that have the same feel and look of gel can be pressed, pushed, pulled, deformed, twisted or even cut into pieces of almost any shape.

\section{vi. Liquid-like}

Liquid or water can also be deformed by poking liquid surface with user's finger, or stroking it with his hand. Scooping some liquid can b a useful interaction technique as well.

\section{2) Non-Deformable Manipulations}

Non-deformable actions or in-hand manipulation skills refers to the ability to shift objects about with the fingers of one or both hands, such as to position an object in some way, move it from one place to another or just change its orientation. In-hand manipulations in [11] depends on mobility and coordinated control at the base joints of the user's fingers, which allow for thumb-finger oppositions in different positions.

According to [11] the three motion types of in-hand manipulations can be classified into three categories: 
International Journal of Ad hoc, Sensor \& Ubiquitous Computing (IJASUC) Vol.4, No.4, August 2013

i. Rotation: is the act of turning something

\section{a. Simple rotation}

For example: pick up a pencil that is oriented across your body and then flip it about in your hand so that it is in the writing position

\section{b. Precision rotation}

involves turning something in the fingertips For example: flip a coin over and over in the fingertips

\section{c. Lateral shift}

movement of the thumb against fingers such as to wind up a small toy, that some people call rotation

ii. Translation: is the movement of objects between palm and fingertips and is also referred to as squirreling (to palm) and de-squirreling (to fingertips) small items.

For example: selecting one coin from several in your palm and moving it to the fingertips with only one hand active.

iii. Shift: is the balanced movement with opposed thumb and finger. For example: thread a needle, push a button through a hole, or poke a pin into a pincushion.

\section{3) Drawing \& Texting}

Free drawing and texting hand manipulations do not imply mouse cursor drawing or keyboard texting, but indicates to the use of bare fingers to type on a graphical or projected on-screen keyboard or draw free-hand letters, numbers, figures or shapes directly on the display surface. One type of free texting is often referred to as touch typing such as thumb typing.

Thumb-texting, for instance, is quite faster than the tradition way of typing on a keyboard input device, once the user gets used to it. Thumb-texting is used widely nowadays in the latest mobile phone touch-based devices that automatically detects when the user desires to type a word, message or phrase, the device produces a graphical on-screen keyboard on which the user uses both his/ her thumb fingers (of both her/ his left and right hands) to type.

\section{4) Tangibles}

Handling tangibles is the basis of TUI interaction techniques, whereas in OUI it is considered one of many hand-based input interaction techniques.

Tangibles are simply graspable physical artifacts that are used to control digital information seamlessly in the user's physical environment. Thus, tangible-based interfaces make use of the already existing usability experience and haptic interaction skills that users have with real-world objects. Not only does tangibles give physical representation to the digital content, but also 
enables this digital content -represented by the physical tangible- to be directed manipulated through the user by his bare hand.

Consequently, using Tangibles are basically natural but non-organic, however, they do can contribute in an organic system in some cases as an aid tool to add more intuition to the system. For example, handwriting with bare fingers can seem more awkward than handwriting with a pen. That is why using a pen stylus in this case will be more natural to the user than his own hands.

A stylus -or a digital pen- is a typical most common type of tangible input tools that provide faster and more accurate input than the older input techniques providing the user with a similar feeling to using an actual pen with great accuracy by detecting the pressure applied with the stylus pen on the device display. Stylus pens are used widely with the latest smart mobile phones and tablet PCs providing users with various input techniques and possibilities that cannot be providing using traditional input techniques or even hand touch or manipulations.

\section{Gestures}

The notation 'Gestures' in this paper is used to mean only 'in-air gestures'. Gestures are the nonverbal form of interaction that relies on visible movements of human hands, face and/or body. Although gestures have been used by man since the beginning of mankind as a primary means of communication that is even more efficient than speech, however it has only been used in the field of human computer interaction just recently.

As gestures are a natural way of communication between human to deliver a certain message, it can easily be used by computer users to issue a command for interaction, once the system is designed to capture hands movements, facial expressions, body motion and eye tracking.

There are different types of gestures that can be used as different input techniques for various sort of commands for performing certain tasks or actions. The first type is 'Single hand gestures', where users can perform using only one hand. Single-hand gestures not only simple and easy, but are natural gestures that users master in their daily life. However, they may differ in their meaning from a country or a culture to another. Examples for single-hand gestures are: still finger(s) and moving finger(s) gestures. Still finger(s) single-hand gestures, include but are not limited to, the 'OK' gesture, the 'victory' gesture, the 'thump-up' gesture, the 'high five', and the 'salute' signs. The other sub-type of single-hand gestures is 'moving finger(s)' such as natural gestures we use for waving, money sign, finger gun, come here, ..etc. Other single-hand gestures can be 'fist gestures', such as boxing fist, fist bump and fist pump. Besides single-hand gestures there is two-handed gestures as well, such as clapping, ribbing, approaching palms or many others. On the other hand eye gestures are very important. Eye tracking systems have recently proven their efficiency of saving power when the user is not 'looking' at them, while activating when the user maintains 'eye contact' with the display of the device. Moreover, face gestures of 'facial expressions' play a great deal of our interaction procedures and are almost unconsciously accompanying most of our daily interactions with each other, ironically even through a phone call, users tend to use their facial expressions to unconsciously represent their feelings. Such facial gestures are a powerful interaction style to be utilized as stand-alone interaction technique or as integrated with another one. For example, saying 'hello' while smiling to the device display can activate the device. This will help mininze system errors that can happen due to false input when the users greets another human in the same room saying hello while not 'looking at' the device. 
International Journal of Ad hoc, Sensor \& Ubiquitous Computing (IJASUC) Vol.4, No.4, August 2013

Factors on which a gesture can be captured and identified:

i. The type of the gesture

a. Single hand gestures

b. Two-handed gestures

c. Eye gestures

d. Face gestures

ii. The axis of the gesture motion

a. Landscape: along the $\mathrm{x}$-axis

b. Portrait: along the y-axis

c. Diagonal: along the z-axis

iii. The direction of the gesture motion

a. Positive: forward, left to right,

b. Negative: backwards, right to left

c. Dual: inwards, outwards

iv. The speed of the gesture motion

Gestural-based interfaces usually uses infrared, colored markers and cameras for image recognition and tracking motions. However, in SoundWave[12] the system uses the Doppler effect to capture gestures, by generating an inaudible tone that gets frequency-shifted when it reflects off moving objects such as the user's hand. Using the speaker and microphone already embedded inside the computer, to sense and capture different user gestures around the device, SoundWave can distinguish the basic input interactions such as: scrolling, single-tap and doubletap, activation, deactivation and rotation. For example, SoundWave uses the walking gesture to automatically put a computer to sleep or wake it up and the Two-Handed Seesaw for rotation.

Using gestures as an input technique must consider that it is totally different than designing a touch-based system. "The wrong way to think about in-air gesturing is 'touch at a distance'[13]. Touch-based systems differentiates the start and end of a touch input by the second the user puts his finger(s) on the display surface and the second he lifts them off the surface. Whereas, gesturebased systems are single-mode systems are always in the ON state, ready to capture any user movement, and has no OFF option. In [13] the author refers to this feature of gesture-based interactive style as the "live mic problem" where the system can capture unintended user gestural input by mistake, such as sneezing, scratching, yawning, responding a sound or any other noninput gestures; that is: false positive gestures.

Designing a gesture-based system should consider false gestures, both positive and negative [13]. False-positive gestures occur when the system detects user's unintended gestures because they resemble some defined gestures. False-negative gestures occur when the system ignores users input gestures that are outside the system's narrow precision constraints. 
International Journal of Ad hoc, Sensor \& Ubiquitous Computing (IJASUC) Vol.4, No.4, August 2013

Gestures are one of the interaction techniques that maintain sterility in medical places, clinics and hospitals, where one infected touch or hand manipulation can cause a lot of problems. Moreover, gestures are very useful interaction style for 'large displays' as they are the most appropriate input actions from a distance than touch or hand manipulation actions. However, touch and air gesture can form together hybrid interactive systems for supporting a lot of applications and functionalities.

On the other hand, gestures fail to be efficient in public places or crowded environments where false gestures, both positive and negative, are captured by the system that as we mentioned is in the 'always-on' single-state. Also, a multiuser system is hard to depend on gesture-based interactions or at least must find recognition scenarios to detect different users, thus gestural input does not adequately support collaborative environments.

\section{THE PROPOSED DESIGN GUIDELINES}

We will focus our design guidelines and principles on the organic interface and its input interaction creating three points of interest: the look, feel and design successively.

In light of the design principles of organic user interfaces for both Vertegaal[3] and Holman[6], in addition to the design guidelines for natural user interfaces for Wigdor and Wixon[13], we proposed three general design principles for organic UI design and other specific design guidelines for each interaction style in the SMaG interaction model (Speech, Manipulation and Gesture).

The three proposed general principles for designing organic user interfaces are covering the three aspects of the design, feel and look:

\section{Natural, intuitive feel}

An OUI must feel as natural and intuitive to the user as using everyday real world objects. Thus designers should think of organic interface as a part of the whole ubiquitous computing environment, designing the system with the most natural interactions capable for human. The natural feel should allow the user to "expect output from the input".

\section{Organic, fluid look}

An OUI must look flexible and malleable (non-planer) as much as it makes it natural to see and use. The look of organic systems includes not only the shape, but the color, and texture as well. Organic devices are bendable, rollable, stretchable, moldable or even wearable. The organic look should be fluid enough to:

- take any shape allows the user to input natural interactions.

- change its shape (or at least contents) as means of visual feedback to users actions.

- Flow between states and never abrupt the user with sudden transitions.

\section{Context-aware, calm design}


International Journal of Ad hoc, Sensor \& Ubiquitous Computing (IJASUC) Vol.4, No.4, August 2013

An OUI must be designed -using the best suitable metaphor- to be ubiquitous and calm (in the background) when not in use and aware of the context of use. In the same context, input actions must always give same responses.

Moreover, we proposed design-specific guidelines for designing organic interfaces for each interaction style in the SMaG interaction model (Speech, Manipulation and Gesture).

The OUI design-specific guidelines are:

\section{A. Speech}

When designing a speech-based organic system, take into consideration the below guidelines:

- Best use:

Speech is used as an input interaction style with large displays, home appliances, medical care places requiring sterility.

- Avoid use:

Speech input is avoided in public places, multiuser systems and dangerous actions to avoid errors of high impact.

- How to use:

Speech is captured either by voice recognition techniques or by sub-vocal speech recognition directly from the user's throat.

\section{B. Hand Touch and Manipulation}

When designing a touch-based organic system, take into consideration the below guidelines:

- Best use:

Hand Manipulations is used as an input interaction style with multiuser systems, home appliances, public places, classrooms, offices, showrooms.

- Avoid use:

Hand Manipulations input is avoided in large displays, and medical care places requiring sterility.

- How to use:

Hand Manipulations is captured either by touch-sensing, or by image or video recognition techniques based on the factors of: the manipulation type (deformational manipulations: bend, roll, squeeze, pinch, ..etc, or non-deformational: rotation, translation, shift), the motion axis (xaxis, y-axis, z-axis), motion speed and the motion direction. Touch is recognized by the factors of 
International Journal of Ad hoc, Sensor \& Ubiquitous Computing (IJASUC) Vol.4, No.4, August 2013

touch type (tip, pad, nail, knuckle), number of simultaneous touch points, motion of the touch or is it just a single tab, direction, shape, pressure, duration, distance, and location on the display.

- Consider to:

1. In $2 \mathrm{D}$ spaces, give at least $15 \mathrm{~mm}$ size -in each direction- for touchable items and at least $5 \mathrm{~mm}$ between them.[13]

2. In 3D spaces, use visual feedback (ex. depth) for all moveable elements to acknowledge successful touch, except in systems that use depth (z-axis) in other purposes.[13]

3. Respond to every contact by immediate feedback with fluid transition with no sudden transitions.

4. Enable basic manipulation (ex: single-finger drag \& flick), direct manipulation rather than controls (remove secondary controls whenever possible), and inertia.

\section{Air Gestures}

When designing a gesture-based organic system, take into consideration the below guidelines:

- Best use:

Air Gestures are used as an input interaction style with large displays, home appliances, medical care places requiring sterility.

- Avoid use:

Air Gesture input is avoided in public places, multiuser systems and dangerous actions to avoid errors of high impact.

- How to use:

Air Gesture is captured either by image or video recognition techniques or by inaudible air frequency shift. Gestures are captured based on the factors of: the gesture type (single hand, twohanded, eye, face, body), the motion axis(x-axis, y-axis, z-axis), the motion direction and speed.

- Consider to:

1. Avoid time-based gestures as it delays expert users.

2. Make it clear how to start, continue and terminate a gesture.

3. Apply the gestures principles for a learnable and safe system (identity, negation, inversion, commutativity)

4. Handle false gestures, both positive and negative.

\section{CONCLUSION AND FUTURE WORK}


International Journal of Ad hoc, Sensor \& Ubiquitous Computing (IJASUC) Vol.4, No.4, August 2013

In this paper we proposed an OUI framework for designing organic systems. We then used our OUI framework to create an interaction model for OUI input styles in which we called the SMaG model, referring to the three main types of organic user-input: speech, manipulations and airgestures. We further examined each interaction style of our proposed SMaG Model, presenting the pros and cons of each of them. Finally, we proposed three main OUI design principles, in addition to some design-specific guidelines for each category of our interaction model for organic user interfaces in ubiquitous environments.

In our future work, we intend to apply our SMaG interaction model on some organic systems that are designed in the proposed OUI framework and based on the design principles and guidelines presented in this research paper.

\section{REFERENCES}

[1] Hiroshi Ishii, "Tangible Bits: Beyond Pixels", Proc. Of the Second International Conference on Tangible and Embedded Interaction (TEI'08), Bonn, Germany, Feb 18-20, 2008.

[2] Roel Vertegaal and Ivan Poupyrev, "Organic Interaction Technologies", Communications of the ACM, Volume 51, No. 6, 26-30 June 2008.

[3] David Holman and Roel Vertegaal, "Organic user interfaces: designing computers in any way, shape, or form". Communications of the ACM, vol. 51, New York, USA, June 2008.

[4] Brygg Ullmer and Hiroshi Ishii, "Emerging Frameworks for Tangible User Interfaces", HumanComputer Interaction in the New Millenium," John M. Carroll, August 2001.

[5] A. Van Dam, "Post-WIMP User Interfaces", Communications of the ACM, 1997.

[6] David Holman, Jonathan Diehl, Thorsten Karrer, Jan Borchers, "Organic User Interfaces", http://hci.rwth-aachen.de/organic, Media Computing Group, RWTH Aachen University, Germany, 2007.

[7] Arnout de Vries, "Multi-Touch Interaction Overview", http://www.tno.nl/nui.

[8] Richard Yonck, "The Age of the Interface", World Future Society, MD, USA, 2010.

[9] Chris Harrison, Julia Schwarz, Scott E. Hudson, "TapSense: Enhancing Finger Interaction on Touch Surfaces", ACM UIST'11, October 16-19, 2011, Santa Barbara, CA, USA, 2011.

[10] Sang-Su Lee Boa Kim, Sohyun Kim Xu Jia, Bopil Jin Daeeop Kim, Eunji Choi Kun-pyo Lee, "How Users Manipulate Deformable Displays as Input Devices", Proc. CHI 2010, ACM New York, USA, 2010.

[11] "In-Hand Manipulation Skills", http://school-ot.com/

[12] Sidhant Gupta et al., "SoundWave: Using the Doppler Effect to Sense Gestures", CHI'12, May 5-10, Austin, Texas, USA, 2012.

[13] Daniel Wigdor and Dennis Wixon, "Brave NUI World: Designing Natural User Interfaces for Touch and Gesture", ISBN 978-0-12-382231-4, 2010. 